\title{
Characterization of a Murine Model of Bioequivalent Bladder Wound Healing and Repair Following Subtotal Cystectomy
}

\author{
Mona Zarifpour, ${ }^{1}$ Karl-Erik Andersson, ${ }^{1}$ Sneha S. Kelkar, ${ }^{1}$ Aaron Mohs, ${ }^{1,2}$ Cathy Mendelsohn, ${ }^{3}$ \\ Kerry Schneider, Frank Marini, ${ }^{3}$ and George J. Christ ${ }^{1,4,5, *}$
}

\begin{abstract}
Previous work demonstrated restoration of a bioequivalent bladder within 8 weeks of removing the majority of the bladder (subtotal cystectomy or STC) in rats. The goal of the present study was to extend our investigations of bladder repair to the murine model, to harness the power of mouse genetics to delineate the cellular and molecular mechanisms responsible for the observed robust bladder regrowth. Female C57 black mice underwent STC, and at 4, 8, and 12 weeks post-STC, bladder repair and function were assessed via cystometry, ex vivo pharmacologic organ bath studies, and $T_{2}$-weighted magnetic resonance imaging (MRI). Histology was also performed to measure bladder wall thickness. We observed a time-dependent increase in bladder capacity (BC) following STC, such that 8 and 12 weeks post-STC, BC and micturition volumes were indistinguishable from those of age-matched non-STC controls and significantly higher than observed at 4 weeks. MRI studies confirmed that bladder volume was indistinguishable within 3 months (11 weeks) post-STC. Additionally, bladders emptied completely at all time points studied (i.e., no increases in residual volume), consistent with functional bladder repair. At 8 and 12 weeks post-STC, there were no significant differences in bladder wall thickness or in the different components (urothelium, lamina propria, or smooth muscle layers) of the bladder wall compared with age-matched control animals. The maximal contractile response to pharmacological activation and electrical field stimulation increased over time in isolated tissue strips from repaired bladders but remained lower at all time points compared with controls. We have established and validated a murine model for the study of de novo organ repair that will allow for further mechanistic studies of this phenomenon after, for example, genetic manipulation.
\end{abstract}

Keywords: bladder; murine; regeneration; repair; subtotal cystectomy; wound healing

\section{Introduction}

The promise of tissue and organ regeneration has captured the imagination of the general public. Regeneration has been observed, characterized, and most extensively studied in invertebrates and lower vertebrates, including the limbs, jaw, tail, nervous system, and heart of the axolotl and the newt. ${ }^{1-4}$ In this regard, adult mammals have a much more restricted repertoire for wound healing, repair, and functional replacement of damaged tissues/organ systems, generally thought to be largely restricted to the epidermis, muscle, bone, and liver. On the contrary, many organs have the capacity to repair or regenerate epithelia and muscle after acute damage. ${ }^{5,6}$ Developing a more high-throughput,

\footnotetext{
${ }^{1}$ Wake Forest Institute for Regenerative Medicine, Winston-Salem, North Carolina.

${ }^{2}$ Department of Pharmaceutical Sciences, University of Nebraska Medical Center, Omaha, Nebraska.

${ }^{3}$ Department of Urology, Pathology and Cell Biology, Genetics and Development, Columbia University, New York, New York.

${ }^{4}$ Department of Biomedical Engineering and Orthopaedic Surgery, University of Virginia, Charlottesville, Virginia.

${ }^{5}$ Laboratory of Regenerative Therapeutics, University of Virginia, Charlottesville, Virginia.
}

*Address correspondence to: George J. Christ, PhD, Department of Biomedical Engineering and Orthopaedic Surgery, University of Virginia, PO Box 800759, Room 1133, MR5, Charlottesville, VA 22908, E-mail: gjc8w@eservices.virginia.edu

(C) Mona Zarifpour et al. 2017; Published by Mary Ann Liebert, Inc. This is an Open Access article distributed under the terms of the Creative Commons Attribution License, which permits unrestricted use, distribution, and reproduction in any medium, provided the original work is properly cited. Mary Ann Liebert, Inc. offers reprint services for those who want to order professionally produced copies of articles published under the Creative Commons Attribution (CC BY) license. To obtain a price quote, email Reprints@liebertpub.com. Please include the article's title or DOI, quantity, and delivery destination in your email. 
genetically tractable mammalian model of bladder regeneration is an important first step toward the identification of molecular targets that could better inform the process of mammalian bladder regeneration. The ultimate goal is to leverage these findings to develop novel and more effective regenerative therapeutics that could dramatically improve the quality of life in patients who suffer, for example, from traumatic bladder injuries, bladder cancer, voiding dysfunction, and/ or bladder pain syndrome.

Current approaches to achieve functional tissue/ organ regeneration have utilized scaffolds, cells, and growth factors, alone or in various combinations, to promote a more favorable in vivo environment for tissue repair, regeneration, or replacement. ${ }^{5,7-19}$ In this regard, Atala et al. created autologous engineered bladder tissues that incorporated urothelial and muscle cells from bladder biopsies seeded on collagen-polyglycolic acid scaffolds to reconstruct the bladder in seven patients with myelomeningocele. ${ }^{20}$ These engineered tissues were implanted with or without omental coverage. Patients reconstructed with engineered bladder tissue created with cell-seeded poly glycolic acid-collagen scaffolds and omental coverage showed increased compliance, decreased end-filling pressures, increased capacities, and longer dry periods over time. ${ }^{20}$ However, as recently noted by Andersson, ${ }^{21}$ despite encouraging proof of concept from the results, the more widespread applications of these technologies await further preclinical investigation.

In that regard, the repair potential of the mammalian bladder has been known for over a century ${ }^{22-29}$ and has been explored in canine, ${ }^{22,29,30}$ rat $^{31-34}$ and also in humans. ${ }^{23,24,35}$ Burmeister et al. ${ }^{34}$ observed de novo repair of bladder in a rat model of subtotal cystectomy (STC) and studied the size and functional characteristics of the observed wound healing response. Urodynamic studies revealed that urine storage and elimination profiles were similar to age-matched control native bladders within 8 weeks of STC, while corresponding histological analysis documented the presence of well-defined layers of urothelium, lamina propria, and detrusor in bladders. ${ }^{34}$ In short, the regrown bladders were histologically and functionally indistinguishable from native agematched bladders (i.e., bioequivalent). A follow-up study by Peyton et al. evaluated molecular mechanisms associated with the robust tissue recovery previously observed. ${ }^{34,36-38}$ In fact, Peyton et al. ${ }^{38}$ established timedependent changes in the location of proliferating cell populations during the early stages of functional rat bladder regrowth, suggesting a role for Shh, Gli-1, and
BMP-4 signaling pathways, which are known regulators of urothelial regeneration homeostasis (Mysorekar [bmp], Shh/Gli, Shin). Taken together, these observations indicate that the rodent bladder is capable of robust wound healing and true multilayer tissue regrowth.

The goal of the present study was to extend these investigations to the murine model. We document that following surgical removal of the majority of the murine bladder, the remaining native bladder tissue undergoes robust wound healing and regrowth, achieving a functionally bioequivalent bladder that reconstitutes all three layers of the bladder wall within 8-12 weeks. Importantly, the urothelium, which is a key functional barrier for normal bladder function, appears to have regenerated efficiently and extends across the luminal surface of the bladder, suggesting that regeneration and repair were efficient despite the large amount of tissue removed. These observations have important implications for future studies of mammalian bladder wound healing and/or repair (regeneration), where the power of murine molecular genetics can be leveraged to provide improved mechanistic insight into the regenerative capacity of the bladder, potentially pointing toward novel therapeutic strategies for bladder repair in larger animal models and humans.

\section{Materials and Methods}

Animals

A total of 52 female C57 black mice weighing 20-22 g underwent STC followed by evaluation with urodynamic and in vitro organ bath studies at 4, 8, or 12 weeks post-STC. Two postoperative animal deaths occurred (3.38\% mortality rate), while two mice were removed from the study because of bladder stone formation. All animal protocols were approved by the Animal Care and Use Committee of the Wake Forest University and carried out with strict adherence to the guidelines set forth.

\section{Trigone-sparing cystectomy}

Animals were anesthetized with 2\% isoflurane chamber induction and maintained with $1-2 \%$ via mask with inhalation and spontaneous breathing. The lower abdomen was shaved, and povidone-iodine solution was applied to the surgical site as an antiseptic. Sterile technique was used throughout each procedure. A lowmidline abdominal incision was made, and the bladder was identified and delivered outside the body (Fig. 1). Two 9-0 Vicryl stay sutures were placed on either 

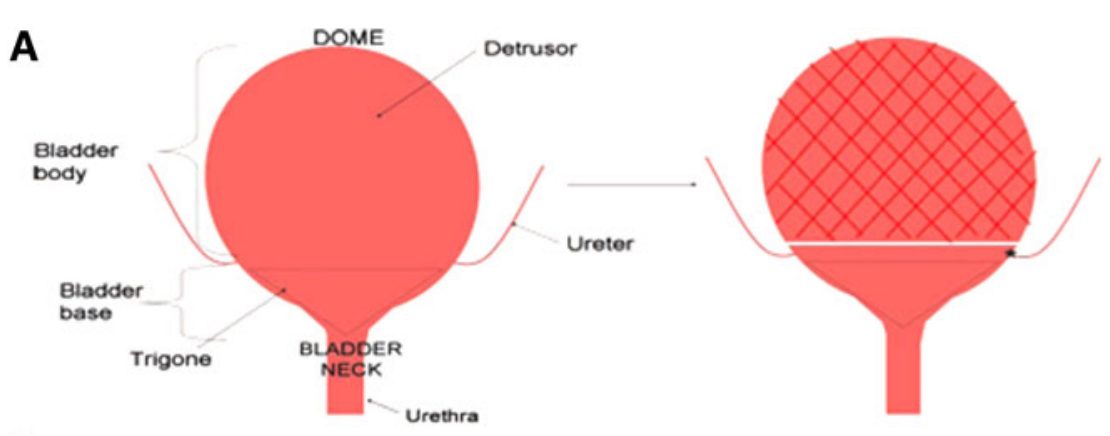

B

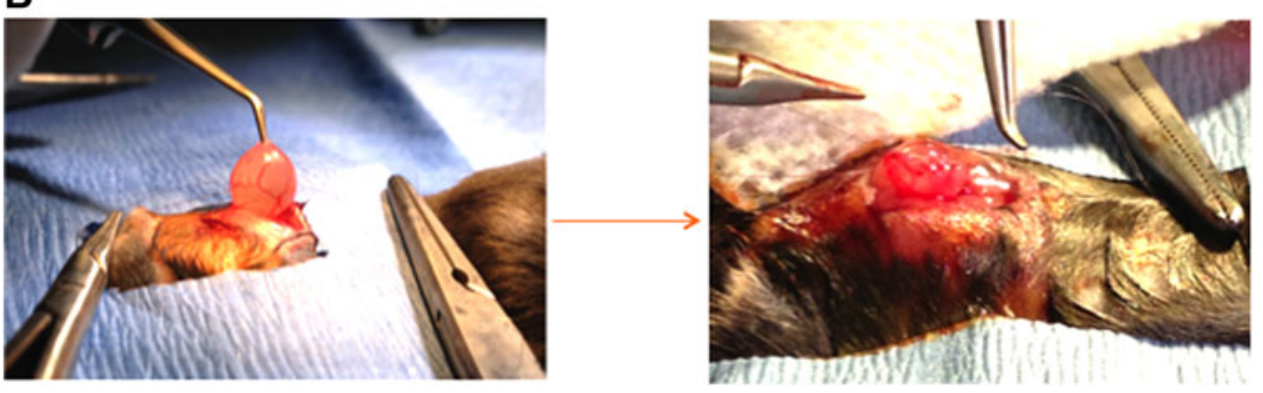

FIG. 1. Subtotal cystectomy (STC). (A) After dissection of the bladder, the bladder body is excised (*50-60\% of the total bladder), leaving the trigone and ureterovesical junction (asterisk) intact. (B) Photographs of STC showing the bladder held in place with stay sutures before excision and after closure.

side of the bladder just above the ureterovesical junction (UVJ). Approximately $60 \%$ of the bladder dome above the UVJ was removed, leaving the trigone and ureters intact (trigone-sparing cystectomy). The remaining portion of the bladder was then closed in two layers with 10-0 (muscularis) and 9-0 Vicryl (serosa) in a continuous manner. The abdominal wall and skin were closed in two layers using 6-0 Vicryl sutures. Animals were followed for up to 12 weeks post-STC.

\section{Bladder catheter implantation}

Urinary catheters were implanted during surgical cystostomy in post-STC and control mice similar to methods previously described. ${ }^{34,39-43}$ Briefly, mice were anesthetized with $2 \%$ isoflurane, and the bladder was dissected from ensuing adhesions caused by the previous surgery. The dome was elevated outside the body as described earlier, a small incision was made by 20 gauge needle, and a PE-10 intramedic polyethylene catheter (Becton-Dickinson) with cuff was inserted and anchored with a 9-0 purse string Vicryl suture. The catheter was then tunneled subcutaneously, brought out through the nape of the animals, and held in place with cloth tape anchored to the skin via a 6-0 Vicryl suture. The abdominal wall and skin were closed in two layers with 6-0 Vicryl sutures, and the free end of the catheter was thermally sealed.

\section{Cystometric analysis}

All cystometric studies were performed at 3 days after catheter implantation in conscious freely moving mice similar to previously described protocols. ${ }^{34,44}$ Briefly, the bladder catheter was connected to a two-way valve that was, in turn, connected to a pressure transducer and an infusion pump. The pressure transducer was connected to an ETH 400 (CD Sciences) transducer amplifier and subsequently connected to a MacLab/8e (Analog Digital Instruments) data acquisition board with accompanying LabChart Pro software. The pressure transducers and acquisition board were calibrated in centimeter of water $\left(\mathrm{cm} \mathrm{H}_{2} \mathrm{O}\right)$ before each experiment. Room temperature saline was infused at a rate of $1.5 \mathrm{~mL} / \mathrm{h}$. Micturition volumes (MVs) were measured with a siliconecoated funnel leading into a collection tube that was connected to a force-displacement transducer. All analyses were conducted after a stable voiding pattern of at least $20-30 \mathrm{~min}$ was established. The terminology and standards for reporting on cystometric analyses follow previous recommendations. ${ }^{44}$ The cystometric 
parameters investigated were basal pressure $\left(\mathrm{P}_{\text {base }}\right.$ lowest pressure between voids), maximum pressure $\left(\mathrm{P}_{\max }\right.$, the maximum pressure seen during a micturition cycle), threshold pressure ( $\mathrm{P}_{\text {thres }}$, pressure at which voiding is initiated), intermicturition pressure $\left(\mathrm{P}_{\mathrm{im}}\right.$, mean bladder pressure between voids), bladder capacity (BC), MV (volume of expelled urine), residual volume (RV: $\mathrm{BC}-\mathrm{MV}$ ), and intercontraction interval (ICI), which is most often defined as the time period between two maximum voiding pressures.

In vitro studies

After cystometric analysis, animals were sacrificed with $\mathrm{CO}_{2}$ inhalation and bilateral thoracotomy, and the bladders were harvested and immediately placed in ice-cold Krebs buffer. The bladders were cut into two strips along the longitudinal axis. The strips were denuded of the urothelium and were attached to tissue holders at one end and force transducers at the other in an organ bath system (Danish Myo Technology) containing $15 \mathrm{~mL}$ of Krebs buffer aerated with 95\% $\mathrm{O}_{2} / 5 \% \mathrm{CO}_{2}$ at $37^{\circ} \mathrm{C}$. Bladder strips were subjected to a resting tension of $400-600 \mathrm{mg}$ and allowed to stabilize for at least $15 \mathrm{~min}$. They were then primed using $60 \mu \mathrm{M} \mathrm{KCl}$ and subsequently $5 \mu \mathrm{M}$ carbachol. Contractions were recorded as changes in tension from baseline in response to both carbachol and electrical field stimulation (EFS). Carbachol concentration-response curves were generated by adding increasing concentrations of carbachol at $0.5 \log$ increments starting at $3 \mathrm{nM}$ up to $100 \mu \mathrm{M}$. For EFS, strips were placed between two platinum electrodes in the organ chamber, and electrical pulses ( $0.1 \mathrm{msec}$ pulse width, $22 \mathrm{~V}$ in the bath) were delivered, lasting $30 \mathrm{sec}$ at increasing frequencies $(1,2$, $4,8,16$, and $32 \mathrm{~Hz}$ ), using an S88 stimulator (Grass Instruments). All tissue responses were normalized to grams of tissue weight.

\section{Magnetic resonance imaging}

A total of three female C57 black mice were evaluated before and after STC. Magnetic resonance imaging (MRI) studies were performed at $0,3,28$, and 77 days after STC. Before MR scans, animals were anesthetized with $3 \%$ isoflurane mixed with oxygen at $2 \mathrm{~L} /$ min flow rate (for induction only). Mice underwent $T_{2}$-weighted MRI with no-contrast agent. All MRI experiments were performed in a $7 \mathrm{~T}$ horizontal bore small animal MRI scanner (Bruker 70/30 Biospec, Billerica, MA) equipped with a high-power $60 \mathrm{~mm}$ I.D. gradient/shim insert capable of producing a maximum magnetic field gradient of $1000 \mathrm{mT} / \mathrm{m}$. A quadrature $35 \mathrm{~mm}$ I.D. volume coil tuned to $300.2 \mathrm{MHz}$ was used for RF transmission and reception. Each animal was placed so that the bladder was at the isocenter of the magnet and RF coil. Anesthesia was maintained during the scan via nose cone, which provided oxygen $(1 \mathrm{~L} /$ min) and isoflurane (1.5\%). Body temperature was kept constant by thermostatically controlled warm air (SA Instruments, Stoney Brook, NY). T2-weighted MR images were acquired using a Rapid Acquisition with Relaxation Enhancement (RARE) spin echo pulse sequence with the following parameters: Repetition time $=9000 \mathrm{msec}$, echo time $=36 \mathrm{msec}$, field of view $=2 \mathrm{~cm}$, matrix $=128 \times 128$, slice thickness $=$ $0.4 \mathrm{~mm}$, number of excitations $=2$. The three-plane localizer verified that the bladder of each mouse was centered in both the RF coil and the magnet. All the data obtained during MRI studies were processed and analyzed with TeraRecon 3D visualization and image analysis software (V4.4.8.36).

\section{Histological analysis and immunohistochemistry}

Bladders not subjected to pharmacological analysis were preserved for histology. After removal of the most superior part of the dome, bladders were fixed in $10 \%$ buffered formalin and processed. Serial $7 \mu \mathrm{m}$ cross sections were sliced along the upper part of the bladder wall (above the uretero-vesical junction). At least six sections were examined from the proximal and distal ends of the bladder. Slides were cleared in xylene, rehydrated, and used for staining. Staining with standard hematoxylin and eosin was performed. For measurement of bladder wall thickness, at least five measurements were taken from three different sections of the bladder as previously described. ${ }^{34}$

For immunostaining, bladders were fixed, embedded in paraffin, and serial sections were generated. Sections were deparaffinized using HistoClear and rehydrated through a series of ethanol and $1 \times$ PBS washes. Antigen retrieval was performed by boiling slides for $30 \mathrm{~min}$ in pH9 buffer. Primary antibodies in $1 \%$ horse serum were incubated overnight at $4^{\circ} \mathrm{C}$. On the next day, slides were washed with TBSTX three times for $10 \mathrm{~min}$ each and secondary antibodies were applied for $2 \mathrm{~h}$ at room temperature. DAPI was applied for $10 \mathrm{~min}$ for nuclear staining and then the slides were cover-slipped.

The following primary antibodies have been applied. For Trp63 staining, we used mouse IgG (clone 4A4, 1:100; Santa Cruz Biotechnology, sc8431) or rabbit 
IgG, (1:100; Santa Cruz Biotechnology, sc8343). For Krt5, we used rabbit IgG (1:300; Covance, AF-138, PRB-160P) or chicken IgY (1:300; Covance, SIG3475). For Upk3, we used mouse IgG1, clone AU1 (1:50; Fitzgerald, 10R-U103a).

\section{Statistical analyses}

Statistical evaluations were performed using GraphPad Prism software. For pharmacological studies, individual carbachol- and EFS-induced stimulus-response curves were placed in GraphPad software and a mean curve was fit to the family of curves. Mean values for the stimulus-response contraction curves were analyzed via one-way analysis of variance (ANOVA). One-way ANOVA was also performed on all relevant parameters from the cystometric studies, as well as the results of histological analysis and MRI measurements. In all cases, a $p$-value of less than 0.05 was considered significant. Unless otherwise stated, all results are expressed as the mean \pm standard error of the mean.

\section{Results}

Mice that successfully recovered from surgery did not show any difference in weight gain $(6.60 \pm 0.610 \mathrm{~g}$, $n=39)$ compared with noncystectomized animals (6.54 $\pm 0.74 \mathrm{~g}, n=13)$.

\section{Cystometric analysis}

Mean values for all urodynamic parameters from cystectomized and age-matched control animals are shown in Table 1. Age-matched controls were treated as one group because no age-dependent differences were noted in any of the cystometric parameters among the three time points in this study $(4,8$, and 12 weeks post-STC). Cystometric investigations showed a progressive increase in $\mathrm{BC}$ at 4,8 , and 12 weeks post-STC (Fig. 2). Moreover, $\mathrm{BC}$ at the 8 - and 12-week time points was not statistically different from controls. The increase in MV also paralleled the increase in BC data, which significantly increased from 4 to 12 weeks and documented that all bladders emptied completely as they regained size post-STC.

Differences in several measures of bladder function were noted in regenerating bladders at the 4 -week time point, relative to bladders from control animals or animals at the 8- and 12-week time points post-STC. For example, there was a significant increase in baseline pressure at 4 weeks compared with controls and 8 weeks post-STC. $\mathrm{P}_{\max }$ generated in animals at 12 weeks post-STC was increased $\left(33.84 \pm 1.27 \mathrm{~cm} \mathrm{H}_{2} \mathrm{O}\right)$ compared with 8 weeks $\left(28.63 \pm 1.06 \mathrm{~cm} \mathrm{H}_{2} \mathrm{O}\right)$ and 4 weeks $\left(29.84 \pm 1.43 \mathrm{~cm} \mathrm{H}_{2} \mathrm{O}\right)$ post-STC. Although $\mathrm{P}_{\max }$ was statistically different at 8 weeks compared with controls, it was not physiologically or clinically relevant and indeed normalized by 12 weeks. Importantly, all bladders at each time point were able to empty completely as evidenced by similar RVs between age-matched control and post-STC animals (Table 1). There was an increase in $\mathrm{P}_{\text {imp }}$ at 4 weeks compared with controls and 8 weeks post-STC. There was no significant difference in $\mathrm{P}_{\text {thres }}$ at any time point post-STC. In addition, statistical analysis also revealed significant differences in the calculated parameter $\left(\mathrm{P}_{\text {thres }} / \mathrm{P}_{\text {max }}\right) . \mathrm{P}_{\text {thres }} / \mathrm{P}_{\max }$ nominally denotes the relationship between the pressure at which micturition is initiated relative to the maximal pressure at which micturition occurs. As indicated in Table 1, $\mathrm{P}_{\text {thres }} / \mathrm{P}_{\max }$ increased significantly at 4 weeks post-STC relative to controls and 12 weeks post-STC. A decrease in ICI was also observed at 4 weeks post-STC compared with controls. ICI at 8 and 12 weeks was not different from controls.

In vitro studies

Steady-state concentration-response curves were obtained for carbachol-induced contractile responses

Table 1. Urodynamic Parameters as Determined by In Vivo Cystometry

\begin{tabular}{|c|c|c|c|c|c|c|c|c|c|}
\hline & $\mathrm{ICI}(\min )$ & $B_{\text {cap }}(m L)$ & $\mathrm{MV}(\mathrm{mL})$ & $\mathrm{RV}(\mathrm{mL})$ & $\begin{array}{c}\mathrm{BP} \\
\left(\mathrm{cm} \mathrm{H} \mathrm{H}_{2} \mathrm{O}\right)\end{array}$ & $\begin{array}{c}\text { TP } \\
\left(\mathrm{cm} \mathrm{H} \mathrm{H}_{2} \mathrm{O}\right)\end{array}$ & $\begin{array}{c}\mathrm{MP} \\
\left(\mathrm{cm} \mathrm{H_{2 }}\right)\end{array}$ & $\begin{array}{c}\text { IMP } \\
\left(\mathrm{cm} \mathrm{H_{2 }} \text { O) }\right.\end{array}$ & TP/MP \\
\hline Controls, $n=10$ & $8.52 \pm 0.48$ & $0.215 \pm 0.013$ & $0.201 \pm 0.01$ & $0.015 \pm 0.008$ & $12.04 \pm 0.90$ & $17.80 \pm 1.22$ & $32.32 \pm 1.23$ & $13.63 \pm 0.86$ & $0.55 \pm 0.02$ \\
\hline 4 weeks, $n=10$ & $5.96 \pm 0.60^{a}$ & $0.149 \pm 0.015^{a, b, c}$ & $0.139 \pm 0.01^{a, b, c}$ & $0.010 \pm 0.006$ & $15.94 \pm 0.98^{a, b}$ & $19.52 \pm 1.20$ & $29.84 \pm 1.43^{c}$ & $17.15 \pm 0.96^{a, b}$ & $0.65 \pm 0.03^{a, c}$ \\
\hline 8 weeks, $n=10$ & $7.66 \pm 0.81$ & $0.191 \pm 0.02$ & $0.183 \pm 0.01$ & $0.009 \pm 0.007$ & $12.10 \pm 0.86$ & $17.30 \pm 0.93$ & $28.63 \pm 1.06^{\mathrm{a}, \mathrm{c}}$ & $13.62 \pm 0.73$ & $0.60 \pm 0.03$ \\
\hline 12 weeks, $n=10$ & $7.89 \pm 0.37$ & $0.197 \pm 0.009$ & $0.212 \pm 0.01$ & $0.010 \pm 0.005$ & $14.13 \pm 0.79$ & $19.03 \pm 1.08$ & $33.84 \pm 1.27$ & $15.91 \pm 0.77$ & $0.56 \pm 0.02$ \\
\hline
\end{tabular}

Age-matched controls revealed no differences and were subsequently grouped together as controls.

${ }^{a}$ Significantly different compared with controls.

bignificantly different compared with 8 weeks.

'Significantly different compared with 12 weeks.

$\mathrm{B}_{\text {cap }}$, bladder capacity; MV, micturition volume; RV, residual volume; BP, basal pressure; TP, threshold pressure; MP, micturition pressure; IMP, intermicturition pressure; $\mathrm{ICl}$, intercontraction interval. 


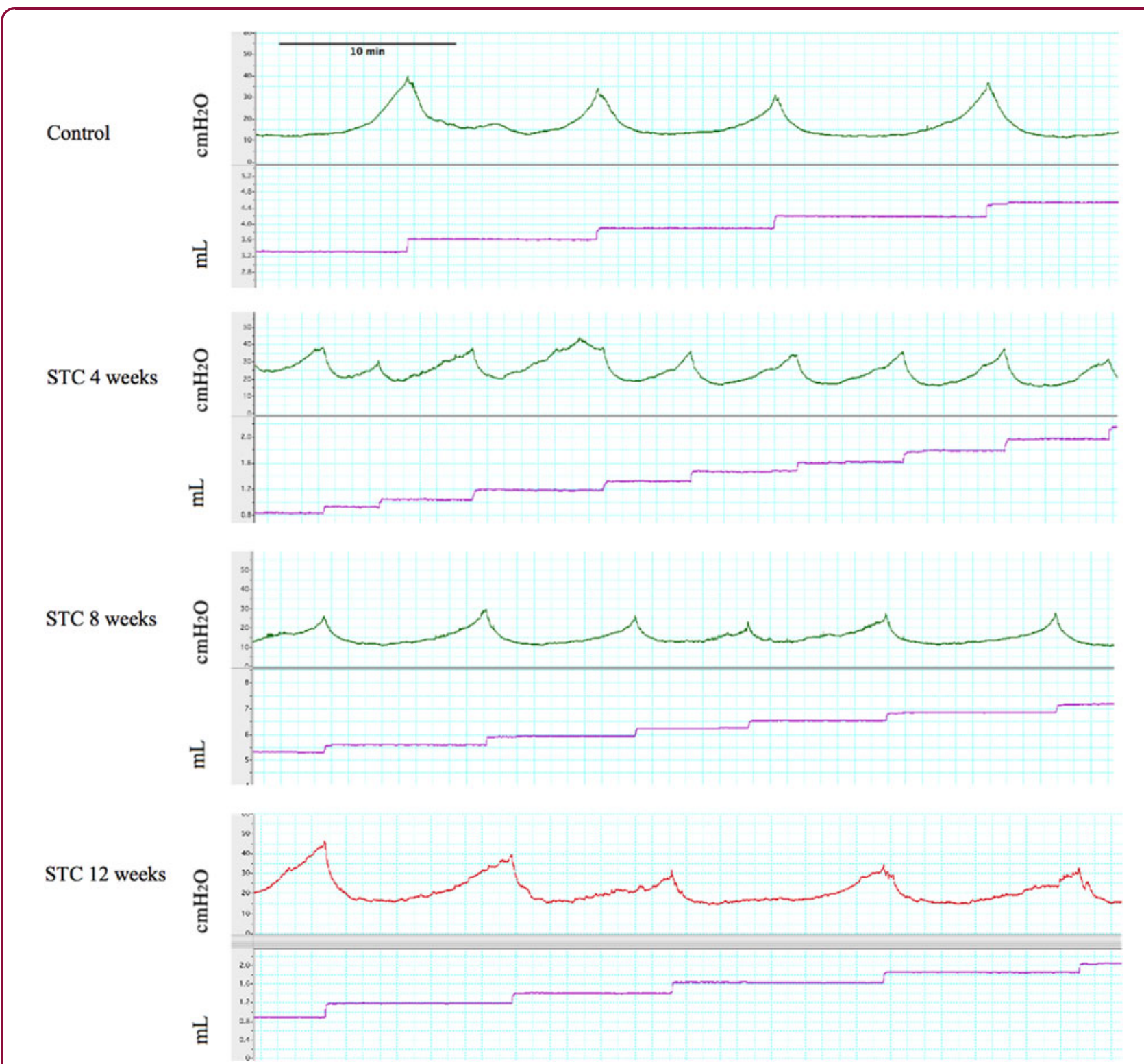

FIG. 2. Representative cystometrogram recordings from control, and 4, 8, and 12 weeks post-STC animals. As can be seen, there was a decrease in intercontraction interval $(\mathrm{ICl})$ at 4 weeks. $\mathrm{ICl}$ at 8 and 12 weeks was not different from controls. Also, there was a decrease in micturition pressure at 4 and 8 weeks, which was normalized at 12 weeks. Scale bar represents $10 \mathrm{~min}$.

in bladder strips from both age-matched control and post-STC animals. Mean values for logistic parameter estimates are shown in Table 2, and the data are depicted in Figure 3. In short, logistic analysis revealed a significant reduction in the calculated maximal steady-state response $\left(\mathrm{E}_{\max }\right)$ values in isolated detrusor strips from the regenerating bladder at all time points (Fig. 3). As illustrated in Figure 3, and summarized in Table 2, there were no de- tectable differences in either $\mathrm{pEC}_{50}$ (the log of the agonist concentration that results in $50 \%$ of the maximum response) or hill slope parameters among the groups. The maximum contractions of regenerating detrusor smooth muscle strips induced by EFS were also significantly lower than those of native tissue at all frequencies tested, and the ratio of native responses to experimental responses was similar across all frequencies (Fig. 4). 
Table 2. Parameters Obtained from Carbachol Dose-Response Curves

\begin{tabular}{lcrr}
\hline & \multicolumn{1}{c}{$\begin{array}{c}\text { E }_{\max } \\
\text { (g/g tissue) }\end{array}$} & $\begin{array}{c}\text { Log [EC } \\
\text { (log[carbachol]) }\end{array}$ & $\begin{array}{c}\text { Hill } \\
\text { slope }\end{array}$ \\
\hline Controls, $n=18$ strips, $n=10$ animals & $140.5 \pm 5.24$ & $-6.009 \pm 0.06$ & $1.63 \pm 0.36$ \\
STC-4 weeks, $n=16$ strips, $n=9$ animals & $85.04 \pm 6.23^{\mathrm{a}}$ & $-5.82 \pm 0.12$ & $1.29 \pm 0.43$ \\
STC-8 weeks, $n=18$ strips, $n=10$ animals & $68.98 \pm 4.50^{\mathrm{a}}$ & $-5.78 \pm 0.11$ & $1.286 \pm 0.38$ \\
STC-12 weeks, $n=14$ strips, $n=9$ animals & $76.54 \pm 6.83^{\mathrm{a}}$ & $-5.91 \pm 015$ & $1.35 \pm 0.60$ \\
\hline
\end{tabular}

There was no difference between groups for either the $\mathrm{EC}_{50}$ or the hill slope values.

${ }^{\mathrm{a}} \mathrm{E}_{\max }$ at all time points is significantly lower than controls $(p<0.05)$.

STC, subtotal cystectomy; $\mathrm{E}_{\max }$ maximal effect; $\mathrm{EC}_{50}$, carbachol concentration producing $50 \%$ of the maximal response.

Magnetic resonance imaging

To study bladder dimensions during the regeneration process, T2-weighted MRI was performed preoperatively, 3 days post-STC, as well as 4 and 11 weeks post-STC (Fig. 5A). The bladder was observed to be close to circular in shape in all cases. At 3 days postSTC, there was a significant reduction in bladder diameter. The overall bladder diameter at day 3 post-STC was $3.65 \pm 0.51 \mathrm{~mm}$, and at weeks 4 post-STC was $4.76 \pm 0.23 \mathrm{~mm}$, and at 11 weeks post-STC was $5.87 \pm$ $0.56 \mathrm{~mm}$ (Fig. 5B). These data are consistent with those from the cystometric analyses as reflected by the parallel increases observed in $\mathrm{BC}$ over the same time frame (Table 1).

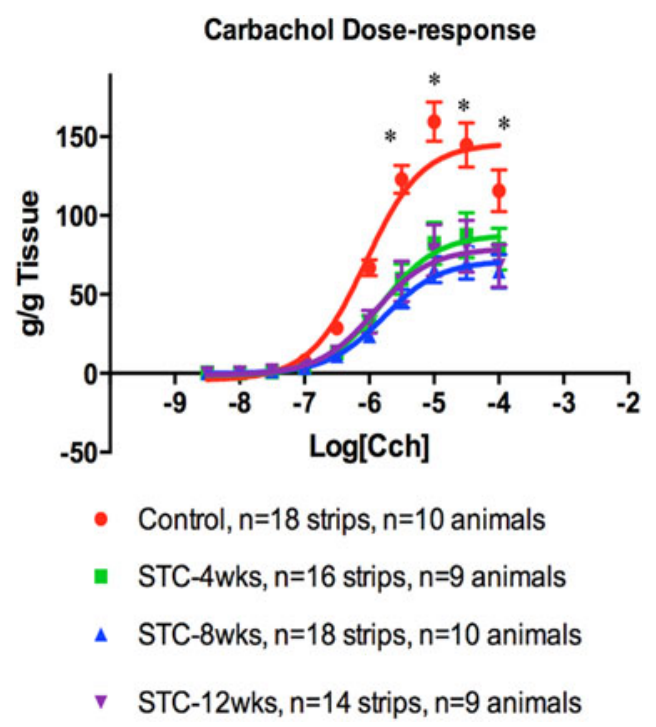

FIG. 3. Carbachol concentration-response curves obtained from bladder strips harvested from control animals, as well as animals at 4, 8, and 12 weeks post-STC. Responses have been normalized to strip weight. ${ }^{*} p<0.05$.
Histological immunochemical analysis

The wall of regenerating bladders appeared to retain the architecture of the native bladder (Fig. 6), as illustrated by the representative examples of hematoxylin and eosin staining of native, 4-, 8-, and 12-week regenerated bladders (Fig. 6A-D). Specifically, the urothelial, suburothelial, and smooth muscle layers appear to be intact in the regenerating bladders. Similar staining of these layers was also seen with trichrome staining (data not shown). Importantly, bladder wall thickness at the 8and 12-week time points $(951.09 \pm 32$ and $1099.065 \pm$ $99.74 \mu \mathrm{m})$ was not significantly different from control bladders $(1096.2 \pm 28 \mu \mathrm{m})$ as shown in Figure 6E. Finally,

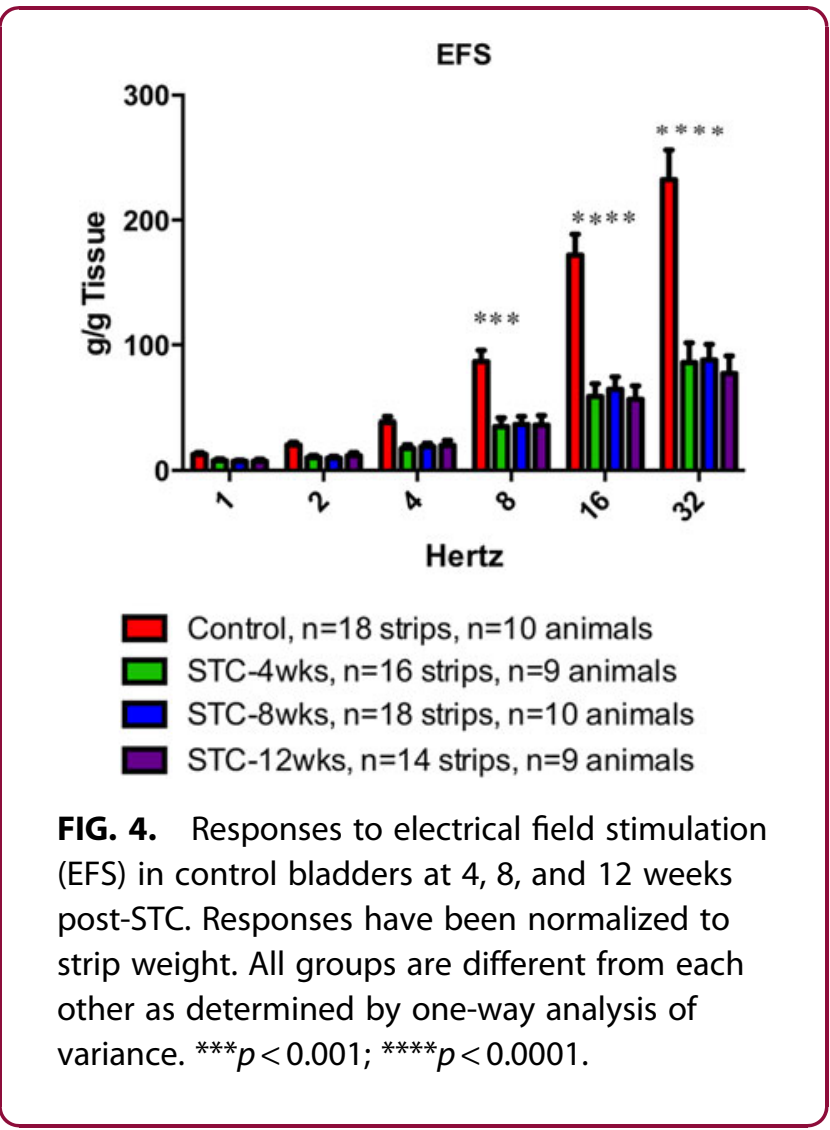




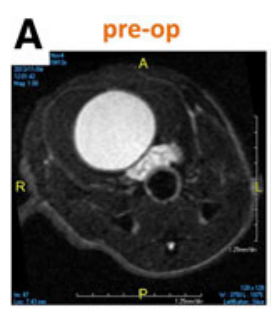

B
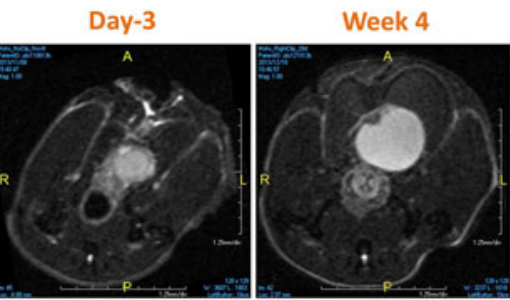

Bladder Diameters from T2-imaging

$$
n=3
$$

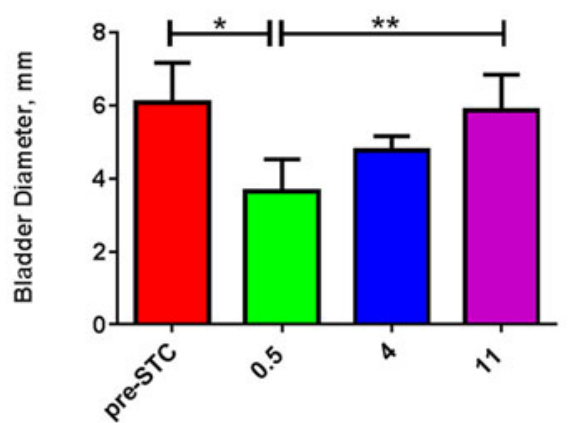

Weeks Post Surgery
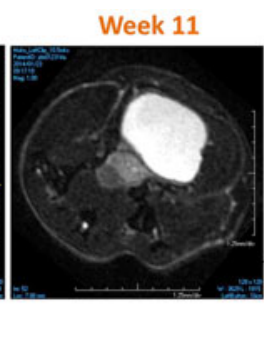

\section{(1)}




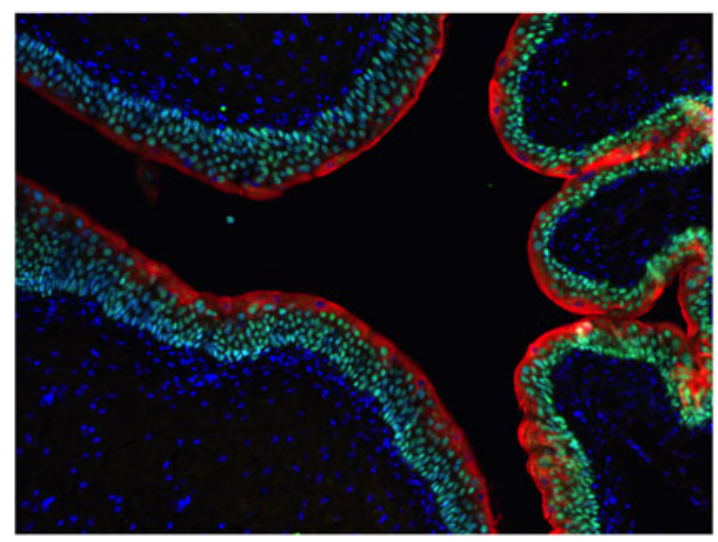

FIG. 7. Representative example of urothelial repair following STC. As illustrated from this animal 12 weeks post-STC, we have observed a remarkable restorative response of the urothelium following removal of $60 \%$ of the murine bladder. Analysis of urothelial markers in bladders 12 weeks after cystectomy showing defined layers, including K5-basal cells (P63+), intermediate cells (P63+/Upk+), and superficial cells (Upk+). Upk expression is shown in red and P63 expression is shown in green. Where p63 (green), Upk1 (uroplakin l; red), and DAPI (blue) in the urothelium. These are all indicative of the complete degree of urothelial bladder repair.

analysis of the urothelium in the bladders of mice 12 weeks post-STC revealed a regenerated urothelium that was virtually indistinguishable compared with unoperated controls (Fig. 7). As expected, the urothelium consisted of 1-2 layers of Krt5-expressing basal cells, intermediate cells, and superficial cells, which were multinucleated and positive for Upk expression.

\section{Discussion}

Consistent with previous observations in rats $^{34}$ the present urodynamic investigation in mice demonstrated a time-dependent increase in BC, with 8-week post-STC mice achieving baseline capacities of age-matched control animals. Bladder capacities increased only slightly from 8 to 12 weeks and were not statistically different from one another. The regrown bladders were no larger than expected for an age-matched control animal at the 12-week time point, suggesting that wound healing and repair response may in some way be dictated by preSTC bladder size. Significantly, bladders in all groups, at all time points, post-STC emptied completely (as indicated by calculated RV [Table 1], even when $\mathrm{P}_{\max }$ was decreased [e.g., 8 weeks]). Moreover, after 4 weeks post-STC, $\mathrm{P}_{\text {thres }} / \mathrm{P}_{\max }$ was similar between all groups. The $\mathrm{P}_{\text {thres }} / \mathrm{P}_{\text {max }}$ parameter describes the relationship between the pressures at the onset of micturition and the maximum achieved during a single void. ${ }^{44}$ Thus, consistency in $\mathrm{P}_{\text {thres }} / \mathrm{P}_{\max }$ between the 8- and 12-week postSTC groups and native (age-matched control) bladders indicates that these bladders are truly bioequivalent from a functional standpoint. Of note, clearly the bladders 4 weeks post-STC, which differed with respect to both bladder volume and pressure, represent a relatively immature and intermediate bladder regrowth stage (Table 1; Figs. 2 and 6). ${ }^{38}$

$\mathrm{KCl}$ - and carbachol-induced contractions were significantly lower at all time points compared with controls, and no significant difference could be detected among the experimental groups. EFS, performed with and without atropine, demonstrated similar effects. While atropine decreased the absolute contractile response in all strips tested, there was no effect on the relative responses of strips from regrown bladders compared with age-matched controls. Importantly, the failure of bladder strips obtained from regrown bladders to achieve contractile responses comparable to agematched controls was in line with other published series in rats. ${ }^{3,36}$ In addition to the decreased amplitude of contraction, a significantly decreased maximal shortening velocity has been demonstrated in the STC bladders, ${ }^{36}$ and it was speculated that this was related to growth and regeneration of muscle cells.

However, there is no definitive explanation for the decreased contractile response since histological investigations revealed an apparently complete reconstitution of all three bladder layers with preserved smooth muscle layer thickness. Functionally, $\mathrm{P}_{\text {thres }}$ and $\mathrm{P}_{\max }$ values were maintained in full-capacity (i.e., $\mathrm{BC}$ equivalent to age-matched control values) regrown bladders that emptied completely at 12 weeks. Thus, the diminished contractile response observed in vitro does not affect the functionality observed in vivo, as evidenced by complete restoration of $\mathrm{BC}, \mathrm{MV}$, and RV. One possibility is that alterations in the orientation of smooth muscle cell bundles in the wall of the repaired/regrown bladder may lead to decreased contractile responses as measured in isolated tissue strips in vitro, without affecting the emptying efficiency of the bladder in vivo. More specifically, since in vitro contractile measurements assess force (grams of tension) largely in the 
longitudinal axis in parallel with the deflection of the force transducer, they would be more sensitive to isotropic alterations in smooth muscle cell orientation than force generation (pressure) measured in vivo (which measures the sum of smooth muscle contractile responses in all orientations in three dimensions).

In short, this mouse model of bladder wound healing, repair, and regrowth post-STC should provide researchers many new opportunities to study the regenerative potential of the mammalian bladder. More specifically, the use of commercially available fluorescent murine models (e.g., green and red fluorescent proteins), coupled to bone marrow irradiation procedures and/or tissue transplants, will permit additional evaluation of the contribution(s) of tissueresident versus systemic precursor cells to bladder wound healing, repair, and regrowth. In conjunction with the use of knockout/knockin transgenic mouse strains of interest, future investigations will be able to identify target cell populations and gene products required for, or associated with, successful bladder reconstitution. This information, in turn, could lead to novel therapeutic (i.e., regenerative) strategies for bladder repair in the face of reductions in viable tissue due to a wide variety of age- and/or disease-related pathologies.

\section{Conclusions}

We have established and validated a murine model for the study of robust bladder wound healing and repair, resulting in regrowth of a bioequivalent bladder within $8-12$ weeks following removal of a majority $(60 \%)$ of the bladder. Future studies in relevant murine models will exploit the power of molecular genetics to further evaluate the cellular and molecular basis of bioequivalent bladder regrowth and thus shed insight on the mechanisms responsible for the apparently marked regenerative potential of the mammalian bladder. The improved mechanistic insight into the regenerative capacity of the bladder should permit the development of novel therapeutic strategies for bladder repair in larger and more clinically relevant animal models and eventually humans.

\section{Author Disclosure Statement}

No competing financial interests exist.

\section{References}

1. Godwin JW, Pinto AR, Rosenthal NA. Macrophages are required for adult salamander limb regeneration. Proc Natl Acad Sci U S A. 2013;110:9415-9420.
2. Petrie TA, Strand NS, Tsung-Yang C, et al. Macrophages modulate adult zebrafish tail fin regeneration. Development. 2014;141:2581-2591.

3. Tsonis PA. Bridging knowledge gaps on the long road to regeneration: classical models meet stem cell manipulation and bioengineering. Mol Interv. 2007;7:249-250.

4. Roy S, Gatien S. Regeneration in axolotls: a model to aim for! Exp Gerontol. 2008;43:968-973.

5. Corona $\mathrm{BT}$, Ward $\mathrm{CL}$, Baker HB, et al. Implantation of in vitro tissue engineered muscle repair constructs and bladder acellular matrices partially restore in vivo skeletal muscle function in a rat model of volumetric muscle loss injury. Tissue Eng Part A. 2014;20:705-715.

6. Pippenger $B E$, Ventura $M$, Pelttari $K$, et al. Bone-forming capacity of adult human nasal chondrocytes. J Cell Mol Med. 2015;19:1390-1399.

7. Corona BT, Machingal MA, Criswell T, et al. Further development of a tissue engineered muscle repair construct in vitro for enhanced functional recovery following implantation in vivo in a murine model of volumetric muscle loss injury. Tissue Eng. Part A. 2012;18:1213-1228.

8. Brown BN, Sicari BM, Badylak SF. Rethinking regenerative medicine: a macrophage-centered approach. Front Immunol. 2014;5:510.

9. De Jong OG, Van Balkom BWM, Schiffelers RM, et al. Extracellular vesicles: potential roles in regenerative medicine. Front Immunol. 2014;5:608.

10. Nauta TD, van Hinsbergh VWM, Koolwijk P. Hypoxic signaling during tissue repair and regenerative medicine. Int J Mol Sci. 2014;15:1979119815.

11. Alberti C. About recent developments of synthetic polymers for a suitable cell adhesion/growth support in tissue engineering-based either augmentation cystoplasty or neobladder. Ann Ital Chir. 2014;85:309-316.

12. Bury MI, Fuller NJ, Meisner JW, et al. The promotion of functional urinary bladder regeneration using anti-inflammatory nanofibers. Biomaterials. 2014;35:9311-9321.

13. Pokrywczynska M, Jundzill A, Adamowicz J, et al. Is the poly (L- lactide- cocaprolactone) nanofibrous membrane suitable for urinary bladder regeneration? PLoS One. 2014;9:e105295.

14. Singh VK, Kalsan M, Kumar N, et al. Induced pluripotent stem cells: applications in regenerative medicine, disease modeling, and drug discovery. Front cell Dev Biol. 2015;3:2.

15. Jia W, Tang $\mathrm{H}, \mathrm{Wu}$ J, et al. Urethral tissue regeneration using collagen scaffold modified with collagen binding VEGF in a beagle model. Biomaterials. 2015;69:45-55.

16. Engelhardt E-M, Micol LA, Houis S, et al. A collagen-poly(lactic acid-co- $\varepsilon-$ caprolactone) hybrid scaffold for bladder tissue regeneration. Biomaterials. 2011;32:3969-3976.

17. Christ GJ. Bladder tissue regeneration. J Urol. 2009:182:16-17.

18. Corona BT, Ward CL, Harrison BS, et al. Regenerative medicine: basic concepts, current status, and future applications. J Investig Med. 2010;58:849-858.

19. Christ GJ, Siriwardane ML, de Coppi P. Engineering muscle tissue for the fetus: getting ready for a strong life. Front Pharmacol. 2015;6:53.

20. Atala A, Bauer SB, Soker S, et al. Tissue-engineered autologous bladders for patients needing cystoplasty. Lancet. 2006;367:1241-1246.

21. Andersson K-E. Words of wisdom. Re: tissue engineering of the bladder: reality or myth? A systematic review. Eur Urol. 2014;66:593.

22. Schwarz R. Ricerche in proposito della rigenerazione della vescica urinaria. Sperimentale. 1981:45:484.

23. Baker R, Tehan T, Kelly T. Regeneration of urinary bladder after subtotal resection for carcinoma. Am. Surg. 1959;25:348-352.

24. Baker R, Maxted WC, Dipasquale N. Regeneration of transitional epithelium of the human bladder after total surgical excision for recurrent, multiple bladder cancer: apparent tumor inhibition. J Urol. 1965;93:593-597.

25. Liang DS. Bladder regeneration following subtotal cystectomy. J Urol. 1962;88:503-505.

26. McCallum DC. Gangrene of the bladder with subsequent regrowth. J Urol. 1965;94:669-670

27. Portilla Sanchez R, Blanco FL, Santamarina A, et al. Vesical regeneration in the human after total cystectomy and implantation of a plastic mould. Br J Urol. 1958;30:180-188.

28. Richardson EJ. Bladder regeneration case report and review of the literature. Minn Med. 1952:35:547-549.

29. Oberpenning F, Meng J, Yoo JJ, et al. De novo reconstitution of a functional mammalian urinary bladder by tissue engineering. Nat Biotechnol. 1999;17:149-155. 
30. Jayo MJ, Jain D, Wagner BJ, et al. Early cellular and stromal responses in regeneration versus repair of a mammalian bladder using autologous cell and biodegradable scaffold technologies. J Urol. 2008;180:392-397.

31. Liang DS, Goss RJ. Regeneration of the bladder after subtotal cystectomy in rats. J Urol. 1963;89:427-430.

32. Liang DS. Secondary cystectomy in rats. J Urol. 1963;90:187-188.

33. Saito $M$, Yoshikawa $Y$, Ohmura $M$, et al. Functional restoration of rat bladder after subtotal cystectomy: in vivo cystometry and in vitro study of whole bladder. Urol Res. 1996;24:171-175.

34. Burmeister D, Aboushwareb T, Tan J, et al. Early stages of in situ bladder regeneration in a rodent model. Tissue Eng Part A. 2010;16:2541-2551.

35. Bohne AW, Urwiller KL. Experience with urinary bladder regeneration. J Urol. 1957;77:725-732.

36. Frederiksen $\mathrm{H}$, Arner A, Malmquist $\mathrm{U}$, et al. Nerve induced responses and force-velocity relations of regenerated detrusor muscle after subtotal cystectomy in the rat. Neurourol Urodyn. 2004;23:159-165.

37. Frederiksen $H$, Davidsson $T$, Gabella $G$, et al. Nerve distribution in rat urinary bladder after incorporation of acellular matrix graft or subtotal cystectomy. Scand J Urol Nephrol. 2008;42:205-212.

38. Peyton CC, Burmeister D, Petersen B, et al. Characterization of the early proliferative response of the rodent bladder to subtotal cystectomy: a unique model of mammalian organ regeneration. PLoS One. 2012;7:e47414.

39. Nomiya M, Burmeister DM, Sawada N, et al. Prophylactic effect of tadalafil on bladder function in a rat model of chronic bladder ischemia. J Urol. 2013;189:754-761.

40. Andersson K-E, Arner A. Urinary bladder contraction and relaxation: physiology and pathophysiology. Physiol Rev. 2004;84:935-986.

41. Christ GJ, Day NS, Santizo C, et al. Bladder instillation of "naked" hSlo/ pcDNA3 ameliorates detrusor hyperactivity in obstructed rats in vivo. Urology. 2001;57(6 Suppl 1):111.

42. Sawada N, Nomiya M, Hood B, et al. Protective effect of a $\beta 3$ adrenoceptor agonist on bladder function in a rat model of chronic bladder ischemia. Eur Urol. 2013;64:664-671.
43. Burmeister DM, AbouShwareb T, Bergman CR, et al. Age-related alterations in regeneration of the urinary bladder after subtotal cystectomy. Am J Pathol. 2013;183:1585-1595.

44. Andersson K-E, Soler R, Füllhase C. Rodent models for urodynamic investigation. Neurourol Urodyn. 2011;30:636-646.

Cite this article as: Zarifpour M, Andersson KE, Kelkar SS, Mohs A, Mendelsohn C, Schneider K, Marini F, Christ GJ (2017) Characterization of a murine model of bioequivalent bladder wound healing and repair following subtotal cystectomy, BioResearch Open Access 6:1, 35-45, DOI: 10.1089/biores.2017.0011.

\section{Abbreviations Used}

ANOVA $=$ analysis of variance

$\mathrm{BC}=$ bladder capacity

BMP-4 = bone morphogenetic protein 4

$\mathrm{EFS}=$ electrical field stimulation

Gli-1 = glioma-associated oncogene

$\mathrm{ICl}=$ intercontraction interval

$\mathrm{Krt5}=$ keratin 5

$\mathrm{MRI}=$ magnetic resonance imaging

$\mathrm{MV}=$ micturition volume

$\mathrm{RV}=$ residual volume

Shh $=$ sonic hedgehog

STC $=$ subtotal cystectomy

Trp63 $=$ transformation-related protein 63

Upk3 = uroplakin III

UVJ $=$ ureterovesical junction

\section{Publish in BioResearch Open Access}

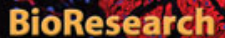

OPEN ACCES oren Access

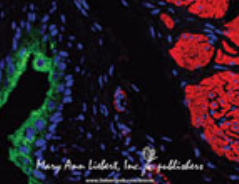

- Broad coverage of biomedical research

- Immediate, unrestricted online access

- Rigorous peer review

- Compliance with open access mandates

- Authors retain copyright

- Highly indexed

- Targeted email marketing 